\title{
THE INFLUENCE OF FILTER PARAMETERS ON THE RESPONSE OF CERAMIC FILTERS OPERATING IN THE THICKNESS-TWIST MODE
}

\author{
S. LEPPÄVUORI and P. RUUSKA \\ Department of Electrical Engineering, University of Oulu, Oulu, Finland.
}

(Received July 3, 1980; in final form November 12, 1980)

\begin{abstract}
Starting with common equivalent circuits, computer programs have been designed to enable the calculation of filter responses from the parameters of the ceramic material and the dimensions of the electrodes. By varying the parameter values their effect on the filter response is shown. To test the calculated results a number of test filters have been made and their responses measured. The filter responses have behaved as predicted by the calculations. It has been possible to calculate the response of thickness-twist mode filters with a centre frequency in the range 1 to $5 \mathrm{MHz}$, a bandwidth of 1 to $10 \%$, a load impedance of 100 to $600 \Omega$ and a comparatively linear phase response.
\end{abstract}

\section{INTRODUCTION}

Ceramic filters intended for the HF range make use of the thickness-vibration resonance mode. There are two principal classes of operation in the thickness mode: Thickness-Dilation (TD) and Thickness-Twist (TT) or Shear (TS). The difference between thicknesstwist and shear-vibration depends on which direction the polarization axis and the electrodes are placed (Figure 1).

Thickness-dilation vibration, where the polarizing axis is at $90^{\circ}$ to the electrodes, is the most commonly used mode of vibration in commercial filters. Filters using thickness-vibration modes have a practical upper frequency limitation which is imposed because it is difficult to machine plates thinner than a half wave-length. By making use of the harmonics of the basic vibration frequency it is possible to produce a filter with a centre frequency in the order of tens of megahertz.

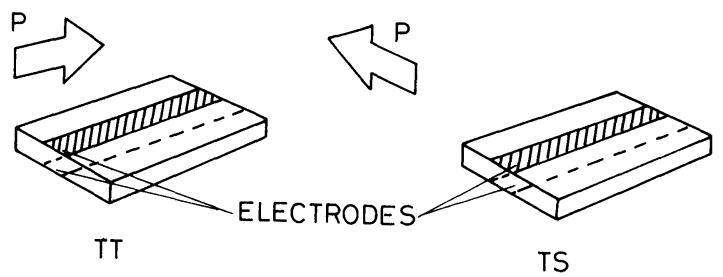

FIGURE 1 The direction of the electrodes and polarization, (P), in thickness-twist, (TT), and shear, (TS), mode vibration.
Thickness-twist mode vibration is theoretically simpler than thickness vibration in the dilation mode and there are no other vibration modes connected with it. Applications of the thickness-twist mode are, however, more limited than the thickness-dilation mode due to a narrower frequency range caused by a small frequency constant and also a high polarizing voltage required during in manufacture. These filters can be used in the frequency range 1 to $5 \mathrm{MHz}$.

The conventional method of making ceramic filters for lower frequencies is to connect single resonators in lattice or ladder networks. Thicknessmode filters are usually constructed in monolithic form by placing more than one resonator on a single plate. This results in a reduction in weight, cost and size and also gives higher reliability. Monolithic filters are based on creating two or more resonances in a single resonator. This also makes it possible to avoid transformers which, in the case of conventional filters, are often required. ${ }^{1,2}$

The creation of more than one resonance is based on the energy trapping phenomenon. By making use of this phenomenon it is possible to form the required number of resonances and also to limit the vibration energy to the area near the electrodes, which is important in the realization of more complicated constructions.

In order to assist the design of ceramic filter operation in the thickness-twist mode, the effect of trapped filter parameters on the filter response has been examined by applying equivalent circuits to the basic element of monolithic filters. 


\section{MATHEMATICAL MODELS OF THICKNESS- TWIST MODE FILTERS AND THEIR COMBINATION}

\subsection{Thickness-Twist Mode Propagation}

Wave movement on a ceramic plate can be propagated only above a certain cut off frequency (waveguide phenomenon). In the electrode part of the plate the cut off frequency is lower than on the part without an electrode. If an electrical vibration which has a frequency between the cut off frequencies is guided onto the electrodes, the vibration reflects off the boundary and the energy is trapped in the vicinity of the electroded region. This results in mechanical and electrical resonances (Figure 2). ${ }^{1}$

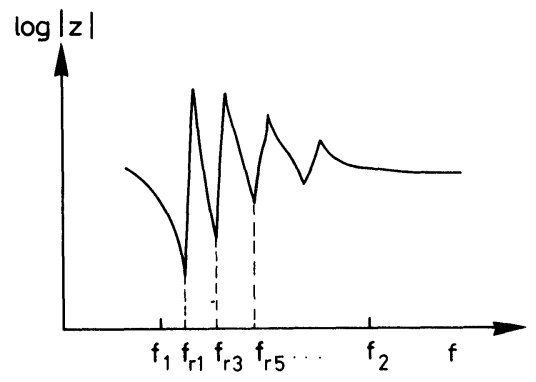

FIGURE 2 Resonances for wide electrodes. $f_{1}$ is the cutoff frequency in the electroded region and $f_{2}$ is the cutoff frequency in the unelectroded region. $f_{r_{2}}$ to $f_{r_{5}}$ are the resonant frequencies.

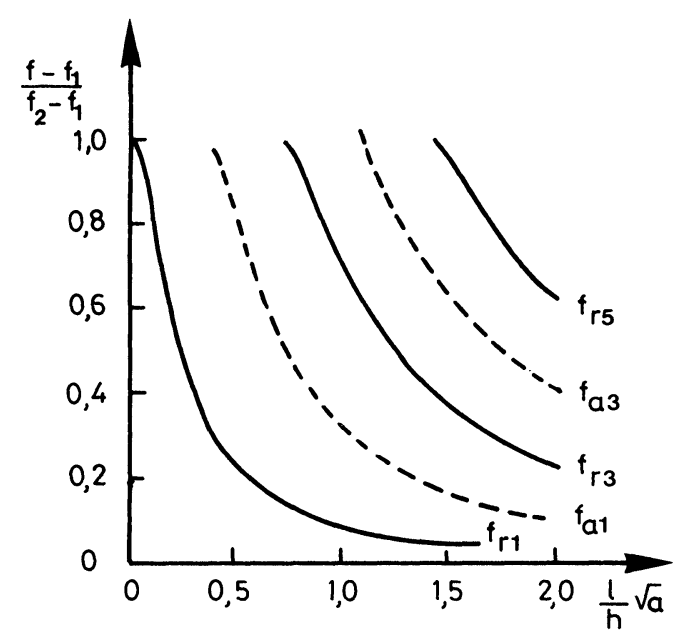

a)
If a ceramic plate is assumed to reach infinity in the $y$-direction and the wave is propagating in the $x$-direction (Figure 3), the upper cut off frequency ${ }^{3}$ is

$$
f_{2}=\frac{1}{2 h} \sqrt{\frac{c_{44}^{C}}{\rho}}=\frac{v_{4}^{D}}{2 h},
$$

where $h$ is the thickness of the ceramic plate, $c_{44}^{D}$ is the elastic shear compliance, $\rho$ is the density of the ceramic material and $v_{4}^{D}$ is the velocity of the vibration propagation (Figure 3). The lower cut off frequency ${ }^{3}$ is

$$
f_{1}=a f_{2}=\xi \frac{h}{\pi} f_{2} .
$$

The wave number, $\xi$, can be calculated from the equations:

$$
\begin{aligned}
& \xi \frac{h}{2} \cot \left(\xi \frac{h}{2}\right)=k_{15}{ }^{2}+R\left(\xi \frac{h}{2}\right)^{2} \\
& R=\frac{2 \rho^{1} h^{1}}{\rho h}
\end{aligned}
$$
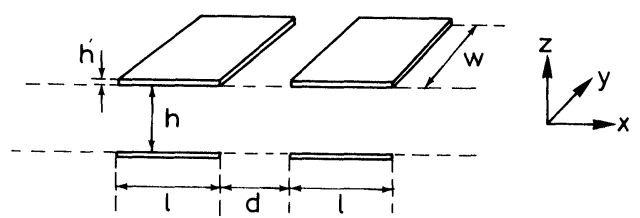

FIGURE 3 The dimensions of the filter.

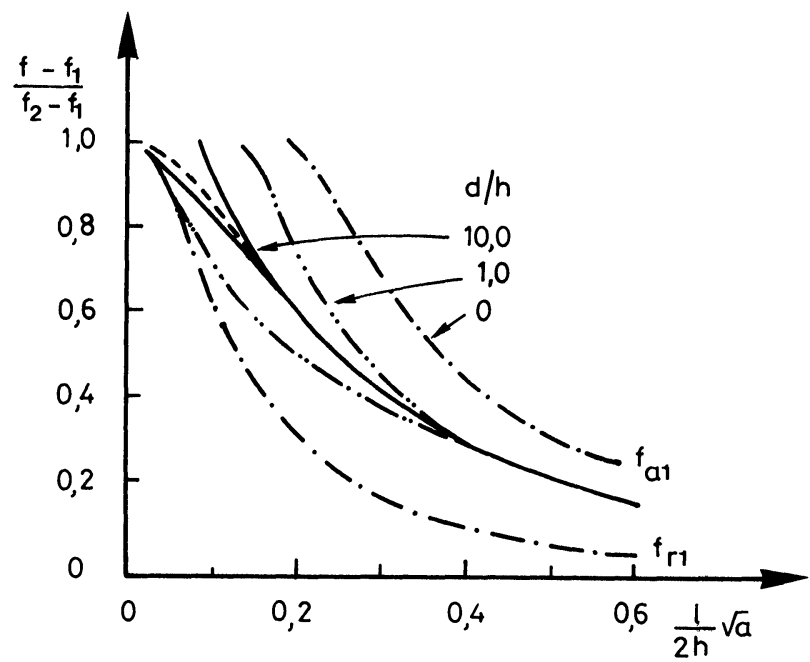

b )

FIGURE 4 a) Resonant frequencies of trapped modes as functions of resonator parameters: - symmetric resonant frequencies, - - antisymmetric resonant frequencies. b) Resonant frequencies of two trapped-energy resonators on the same plate as functions of resonator parameters. The distance between the resonators is $d, h$ is the thickness of the ceramic plate and $l$ is the electrode width (Figure 3 ). 
where $k$ is the electromechanical coupling factor of the thickness-twist mode, $\rho$ ' is the density of the electrode material and $h^{\prime}$ is the thickness of the electrode.

If two resonators are placed on one plate an acoustic coupling exists between them which results in the excitation of antisymmetrical resonances, $f_{a 1}, f_{a 2}, \ldots$, in addition to the basic symmetrical resonances, $f_{r 1}, f_{r 2} \ldots$, (Figure $\left.4 \mathrm{a}\right)$. The basic resonant frequency is split into two resonant frequencies as the two resonators are brought closer to each other as shown in Figure $4 \mathrm{~b}$.

The basic element of a monolithic filter is shown in Figure 5. The dimensions are chosen so that two resonances can exist. The construction where the upper electrode is divided is the equivalent of two closely placed resonators. The double mode resonator acts as a filter whose bandwidth and centre frequency are dependant on the mechanical dimensions.
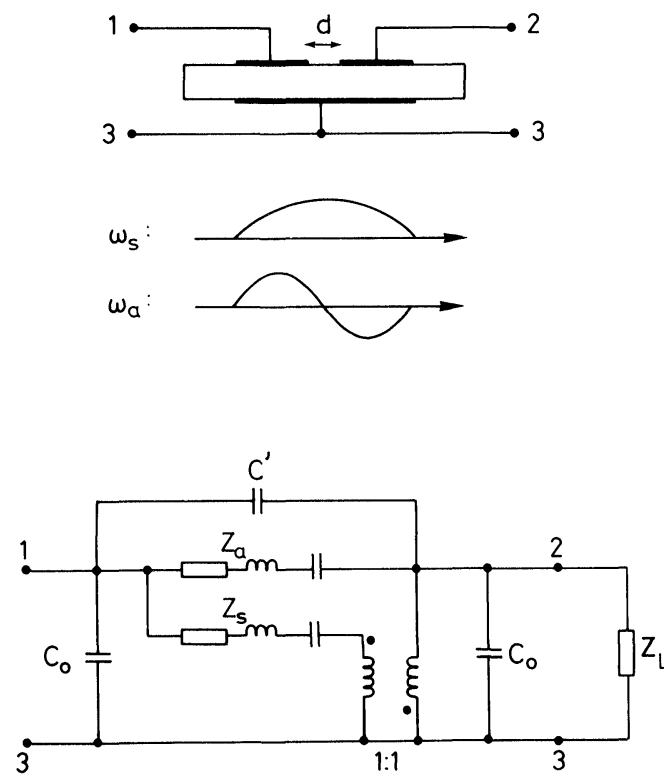

FIGURE 5 The basic element of a monolithic filter, the standing wave corresponding to a symmetric and antisymmetric resonance and the equivalent circuit of the filter.

\subsection{The Onoe Model}

The basic element of a monolithic filter can be described with an equivalent circuit presented by M. Onoe et al (Figure 5). In this model resonators are represented by series resonance circuits, $Z_{a}$ and $Z_{s}$, the capacitances between all the electrodes by
$C_{0}$ and $C^{\prime}$, the phase shift which takes place in the filter by a transformer and the load impedance by $Z_{L}$. The circuit acts as a bandpass filter since only vibrations which have a frequency near the resonant frequency can be passed. The capacitance values of the Onoe model can be calculated from the resonant and antiresonant frequencies of the filters impedance versus frequency plot (Figure 6):

$$
\begin{aligned}
& C_{0}+\frac{2 C_{s}}{1-\left(\omega_{3} / \omega_{1}\right)^{2}}=0 \\
& C_{0}+2 C^{\prime}+\frac{2 C_{a}}{1-\left(\omega_{4} / \omega_{1}\right)^{2}}=0 \\
& C_{0}+C^{\prime}+\frac{C_{s}}{1-\left(\omega_{5} / \omega_{1}\right)^{2}}+\frac{C_{a}}{1-\left(\omega_{5} / \omega_{2}\right)^{2}}=0 \\
& C_{0}+C^{\prime}+\frac{C_{s}}{1-\left(\omega_{6} / \omega_{1}\right)^{2}}+\frac{C_{a}}{1-\left(\omega_{6} / \omega_{2}\right)^{2}}=0
\end{aligned}
$$

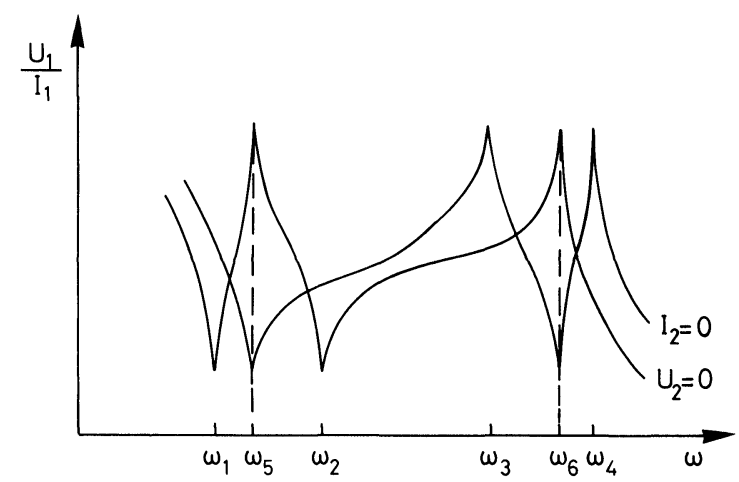

FIGURE 6 The impedance curve of the filter with output open and short-circuited $\left(\omega_{1}=2 \pi f_{r}\right.$, and $\left.\omega_{2}=2 \pi f_{r_{2}}\right)$.

If the $Q$-factor of the resonant circuits is known, the inductances and resistances of the equivalent resonance circuits can be calculated from the capacitance values:

$$
\begin{aligned}
& \omega_{1}^{2} L_{s} C_{s}=1 \\
& \omega_{2}^{2} L_{a} C_{a}=1 \\
& Q=\omega_{1} L_{s} / R_{s} \\
& Q=\omega_{2} L_{a} / R_{a}
\end{aligned}
$$

The values of the static capacitances can also be calculated from the filter geometry with the help of the values in Figure $3 .^{4}$ 


$$
\begin{aligned}
& C_{0}=\epsilon_{0} \epsilon_{11}^{s} w \frac{l}{h} \\
& C^{\prime}=\epsilon_{0} \epsilon_{11}^{s} w\left(\frac{1}{2} \frac{K\left(m^{\prime}\right)}{K(m)}\right)
\end{aligned}
$$

where

$$
\begin{aligned}
& m=\frac{\tanh (\pi d / 4 h)}{\tanh (\pi 2 l+d) / 4 h)} \\
& m^{\prime}=\sqrt{1-m^{2}}
\end{aligned}
$$

$K(m)$ is the elliptic integral of the second kind.

In the equations $\epsilon_{0}$ is the vacuum permittivity and $\epsilon_{11}^{S}$ the relative permittivity of the ceramic material.

Unfortunately the component values of the Onoe model resonance circuits cannot be calculated without first measuring the filter so its use alone when designing filters is not possible.

\subsection{The Nakamura-Shimizu model}

The component values for the resonant circuits of the Onoe model can be obtained by applying and analysing the filter with the Nakamura-Shimizu model, which is based on a physical analysis of the mechanical vibration (Figure 7). In the case of the thickness twist model: $:^{5}$

$$
\begin{aligned}
& C_{0}=w l \epsilon_{11}^{s} / h \\
& N=w l \epsilon_{15} \frac{\sin \xi h / 2}{h / 2} \\
& Z_{e}=w_{2}^{h} c_{44}^{D} \frac{\eta_{e}}{\omega} \\
& \theta_{e}=\eta_{e} l \\
& \eta_{e}^{2}=\frac{\omega 2}{v_{s}^{2}}-\xi^{2} \\
& v_{s}=\sqrt{c_{44}^{D} / \rho} \\
& Z_{s}=w_{\frac{h}{2}} c_{44}^{D} \eta_{s} / \omega \\
& \theta_{s}=\eta_{s} d \\
& \eta_{s}^{2}=\frac{\pi^{2}}{h^{2}}-\frac{\omega^{2}}{v_{s}^{2}}
\end{aligned}
$$

In the equations:

$N$ is the transformation relation between the electrical and mechanical vibrations
$Z_{e}$ is the wave impedance in the electroded region

$\theta_{e}$ is the phase shift in the electroded region

$\eta_{e}$ is the wave number in the $x$-direction, in the electroded region

$\eta_{s}$ is the wave number in the $x$-direction, in the unelectroded region

$\xi$ is the wave number in the $z$-direction

$v_{s}$ is the propagation velocity in the unelectroded region

$Z_{s}$ is the wave impedance in the unelectroded region

$\theta_{s}$ is the phase shift in the unelectroded region

The model can also be applied in the thickness shear mode.

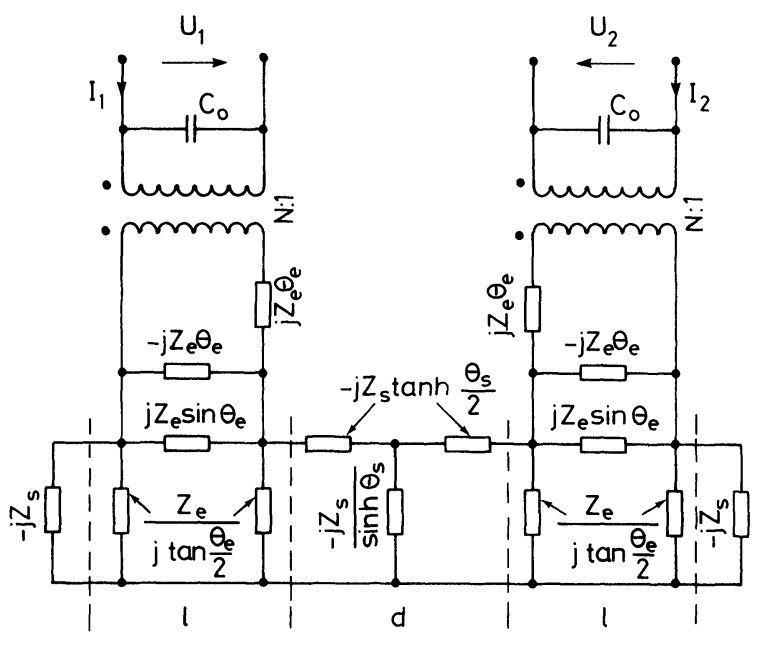

FIGURE 7 The Nakamura-Shimizu-model.

\subsection{Combining the Models}

It is possible to calculate filter responses with the Nakamura-Shimizu model but to be able to use an equivalent circuit with lumped elements to analyze the circuit, the two models of the filter must be combined together. This is done by obtaining the component values of the Onoe model's resonant circuits from the Nakamura-Shimizu model (Figure 8).

The filter admittance at the resonant frequencies is

$$
\frac{1}{Z_{1,2}}=j \omega C_{0}+\frac{N^{2}}{Z_{\mathrm{mek}_{1,2}}},
$$

where $Z_{1}$ is the impedance at the symmetrical resonant frequency and $Z_{2}$ is the impedance at the antisymmetrical resonant frequency. The mechanical 


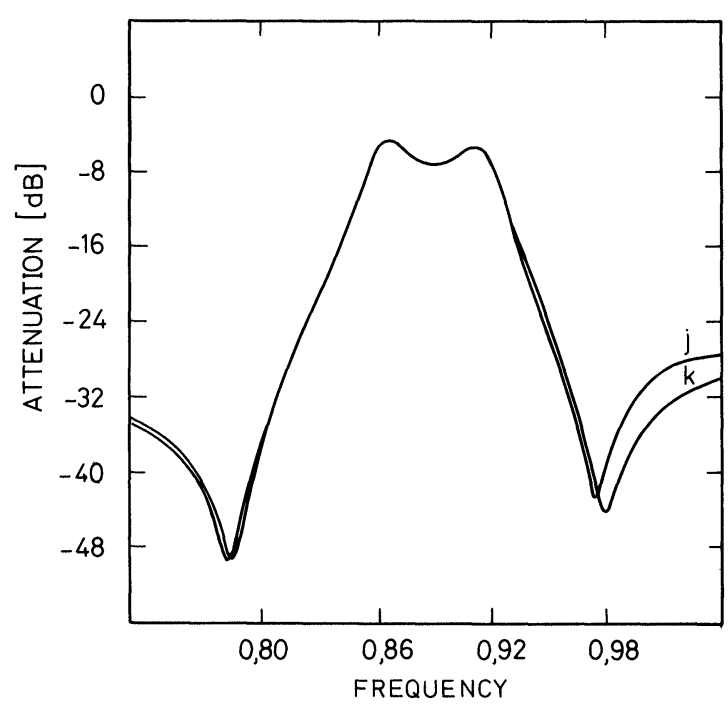

FIGURE 8 Filter response calculated from the NakamuraShimizu $(j)$ model alone and from the Onoe model $(k)$ using the NS model to calculate the lumped elements of the resonant circuits. The frequency is normalized to $f_{2}$.

impedance, $Z_{\mathrm{mek}}$, is derived from ${ }^{6}$

$$
\left.\left.\frac{Z_{\mathrm{mek}_{1,2}}=j Z_{e} \theta_{e}\left(1+\frac{\theta_{e}}{\sin \theta_{e}}-1-\right.}{\left(\tan \frac{\theta_{e}}{2}+\frac{Z_{e}}{Z_{s}}\right)^{-1}+\left(\tan \frac{\theta_{e}}{2}+\frac{Z_{e}}{Z_{s}}\left(\tanh \frac{\theta_{s}}{2}\right)^{-c}\right)^{-1}}\right)^{-1}\right)
$$

where $c=1$ for the symmetric case

$$
c=-1 \text { for the antisymmetric case. }
$$

The other components of the resonant circuits can be obtained from the resonant frequencies and inductances.

\subsection{Computer Program}

The resonant frequencies and the corresponding inductances were calculated from the previous equation with the help of a Fortran program which was based on a stepping method. The program gave the resonant frequencies and inductances and the required width of the electrodes, the distance between them and the constant, $a$, as input data. In order to calculate the filter responses, a program was designed based on the Onoe model. This program calculated the poles and zeros of the filter transfer function as well as the amplitude and phase response of the filter. It required as input data the resonant frequencies, the inductances of the resonant circuits, the quality factor, $Q$, of the filter, the capacitance between the upper electrodes and the load admittance value. In order to compare the two models of the filter, a program was designed according to the NS model to calculate the filter response from the input information. It is possible to link the program, which calculates the resonant frequencies and the inductances as a subprogram, with the common circuit analysis programs. With this linking it is possible to do a more sophisticated analysis on the behaviour of the circuit.

\section{THE INFLUENCE OF PARAMETERS ON THE FILTER RESPONSE}

\subsection{Calculated Results}

Figure 9 shows the effect of the width, $l$, and the distance, $d$, between the electrodes on the number and placement of resonant frequencies. The calculations have been made for the PZT-7A material (Table I). The centre frequency is determined by the thickness, $h$, of the ceramic plate. The width of the electrodes must be decided so that the relation $l / h$

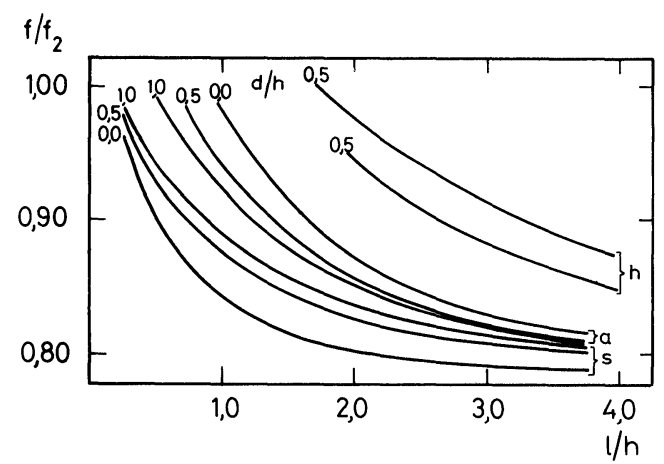

FIGURE 9 The shift of resonant frequencies as a function of the resonator parameters. $h$ is the first harmonic of the basic resonance frequencies.

TABLE I

Properties of PZT-7A material. ${ }^{7}$

\begin{tabular}{lc}
\hline Frequency constant N (TT, TS-mode) & $1250 \mathrm{~Hz} \mathrm{~m}$ \\
$V_{4}^{D}$ & $2490 \mathrm{~m} / \mathrm{s}$ \\
$a$ & 0.775 \\
$\rho$ & $7.6 \cdot 10^{3} \mathrm{~kg} / \mathrm{m}^{3}$ \\
$k_{15}$ & 0.67 \\
$\epsilon_{11}^{S} / \epsilon_{0}$ & 460 \\
$Q_{m}$ & 600 \\
$Q_{e}$ & 60 \\
Curie temperature & $350^{\circ} \mathrm{C}$ \\
\hline The time dependence of $N$ & $-0.08 \% /$ decade \\
The temperature dependence of $N$ & $2.9 \%$ \\
for the temperature range $-60^{\circ} \mathrm{C}$ to $+85^{\circ} \mathrm{C}$ \\
\hline
\end{tabular}




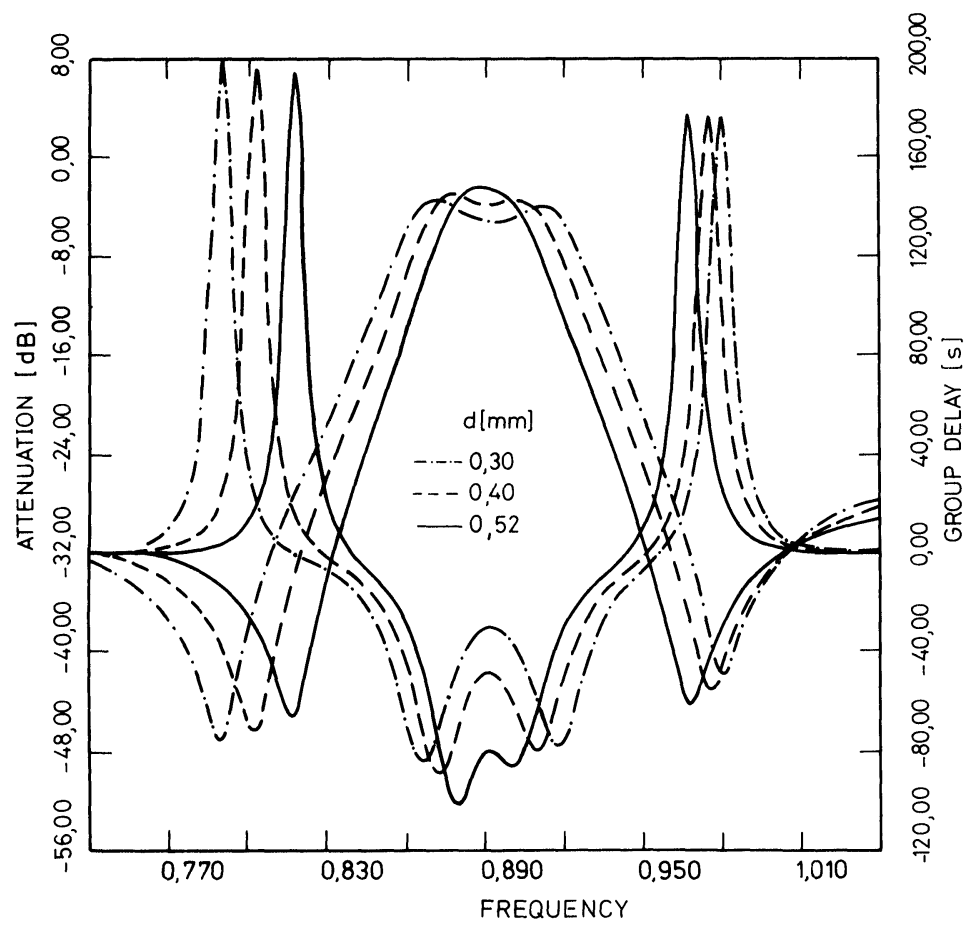

FIGURE 10 Filter responses with different electrode distances.

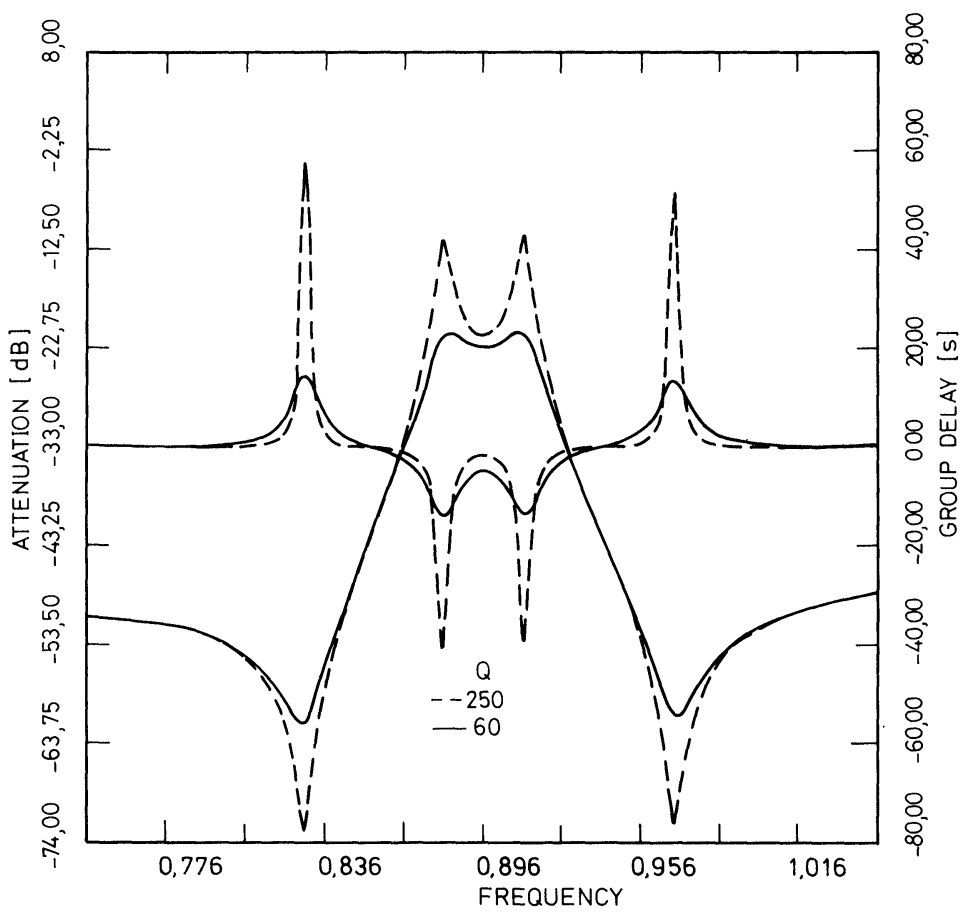

FIGURE 11 Filter responses with $Q$-factors of 60 and 250 . 
has a value which allows two resonances to exist. Figure 10 shows the filter response as the distance between the upper electrodes is varied. It can be seen that the passband width, the ripple and the linearity of the phase response are all dependent on the distance $d$.

The $Q$-factor of the filter affects the height of the resonances, the attenuation, the passband ripple and the phase linearity (Figure 11). The character of the phase response can be seen on the group-delay plot. The magnitude of the $Q$-factor depends on the ceramic material used and can also be effected if covered with an energy absorbing material which is necessary in order to reduce spurious responses in the resonator. It is therefore possible only to estimate the $Q$-factor of the final filter at the design stage. The shifting of the dominant poles of the transfer function in the s-plane, as the load impedance changes, is shown in Figure 12. As the load resistance decreases, the poles move closer to the imaginary axis and their real parts become almost equal (Figure 13). This means that the amplitude response tends to become more symmetrical, the phase response becomes nonlinear, the resonant peaks become sharper and the passband attenuation

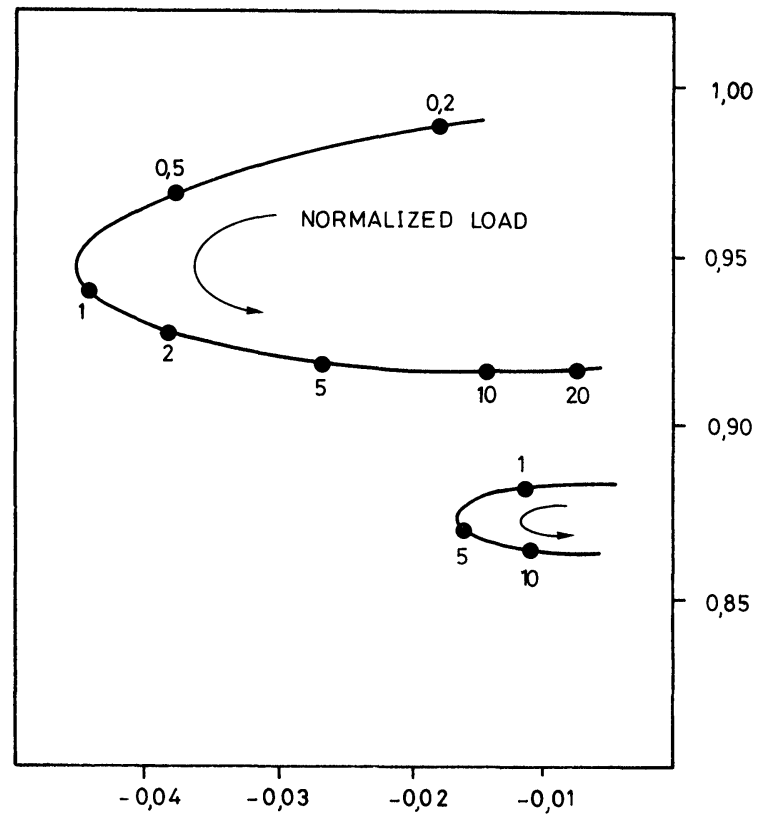

FIGURE 12 The shift of the dominant poles of the transfer function in the $s$-plane as the load admittance changes. The value of the normalized load conductance is shown in the picture.

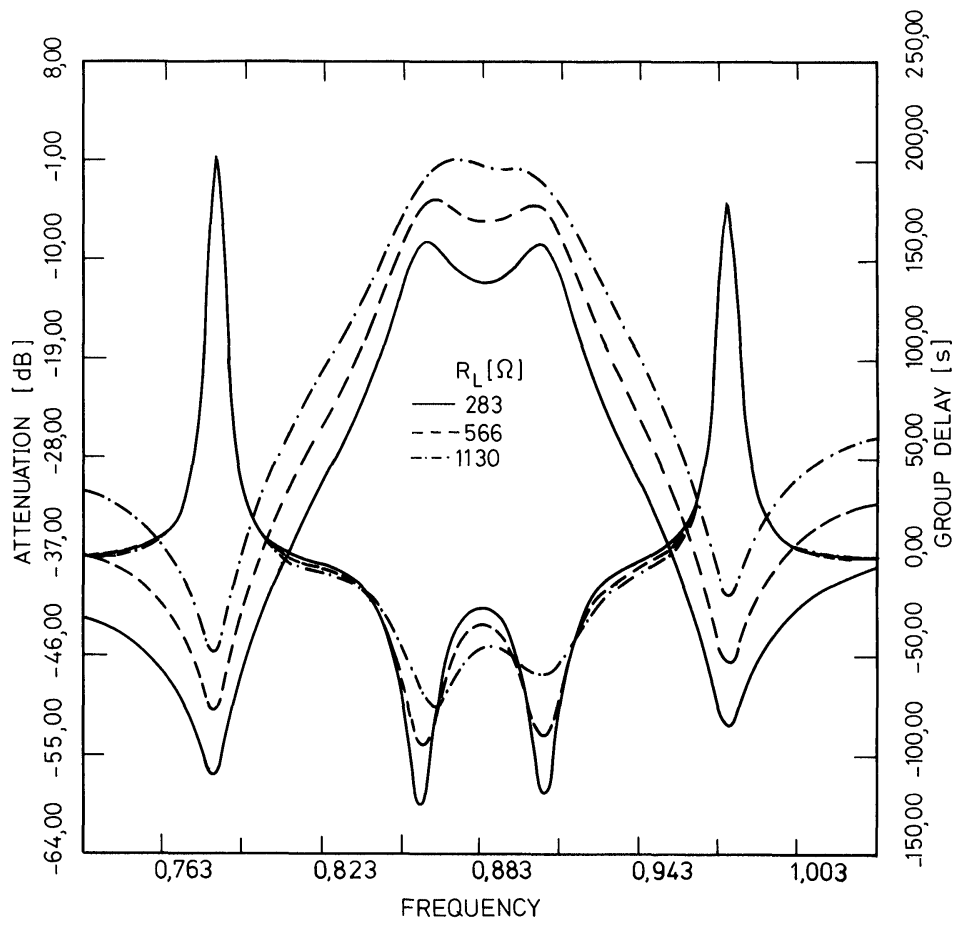

FIGURE 13 Filter responses with different load resistance $R_{L}$. 


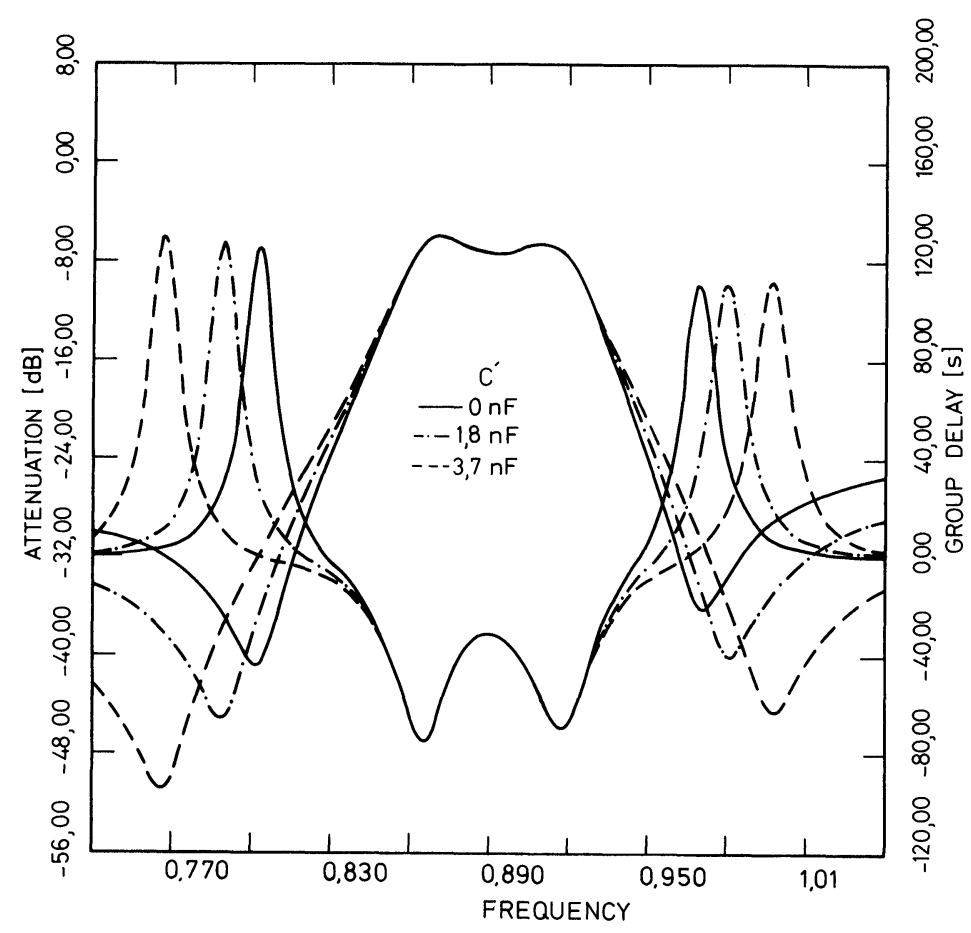

FIGURE 14 Filter responses with different shunt capacitance $C^{\prime}$.

increases. The capacitive part of the load impedance does not have a large effect on the filter response so its value can be ignored.

The placement of the zeros of the transfer function are determined by the capacitance $C^{\prime}$, between the upper electrodes. This value can be made larger by connecting a shunt capacitor across the filter. By changing the capacitance value it is possible to alter the slope of the filter (Figure 14).

\subsection{Experimental Results}

In order to test the calculated results a number of filters were constructed from a commercially

TABLE II

The symmetric, $f_{r 1}$, and antisymmetric, $f_{a_{1}}$, resonant frequencies according to measurements and calculations as the electrode width and the distance between them varies (Figure 3).

\begin{tabular}{lllllll}
\hline & & \multicolumn{2}{c}{ Measured values } & & \multicolumn{2}{c}{ calculated values } \\
\cline { 6 - 7 }$l / h$ & $d / h$ & $f_{r_{1}}$ & $f_{a_{1}}$ & & $f_{r_{1}}$ & $f_{a_{1}}$ \\
\hline 1.21 & 0.57 & 0.842 & 0.878 & 0.863 & 0.915 \\
1.29 & 0.60 & 0.865 & 0.898 & 0.860 & 0.918 \\
1.38 & 1.00 & 0.861 & 0.877 & 0.863 & 0.889 \\
1.53 & 0.71 & 0.850 & 0.876 & 0.847 & 0.881 \\
\hline
\end{tabular}

available material, PZT-7A (Table I). The electrodes were prepared by etching an evaporated layer.

Table II shows the measured resonant frequencies of the filters compared with those of the corresponding calculated values. The resulting differences can be explained by inaccuracies in measuring the thickness of the ceramic plate and in determining the resonant frequencies.

Table III shows the effect of the load impedance on the lower resonant frequency according to the

TABLE III

The symmetric resonant frequency of the filter according to measurements and calculations with different load conductances (PZT-7 A, $h=0.62 \mathrm{~mm}, d=0.35 \mathrm{~mm}$, $l=0.75 \mathrm{~mm}, Q=55$ ).

\begin{tabular}{lll}
\hline $\mathrm{G}_{\mathrm{L}}$ & $f_{m}$ & $f_{c}$ \\
\hline$\infty$ & 0.8609 & 0.8625 \\
43 & 0.8620 & 0.8626 \\
29 & 0.8630 & 0.8627 \\
17 & 0.8635 & 0.8633 \\
12 & 0.8660 & 0.8641 \\
9 & 0.8696 & 0.8696 \\
4 & 0.8742 & 0.8748 \\
0 & 0.8762 & 0.8828 \\
\hline
\end{tabular}

$\mathrm{G}_{\mathrm{L}}$ is the normalized load admittance, $f_{m}$ is the measured resonant frequency and $f_{c}$ is the calculated resonant frequency. 


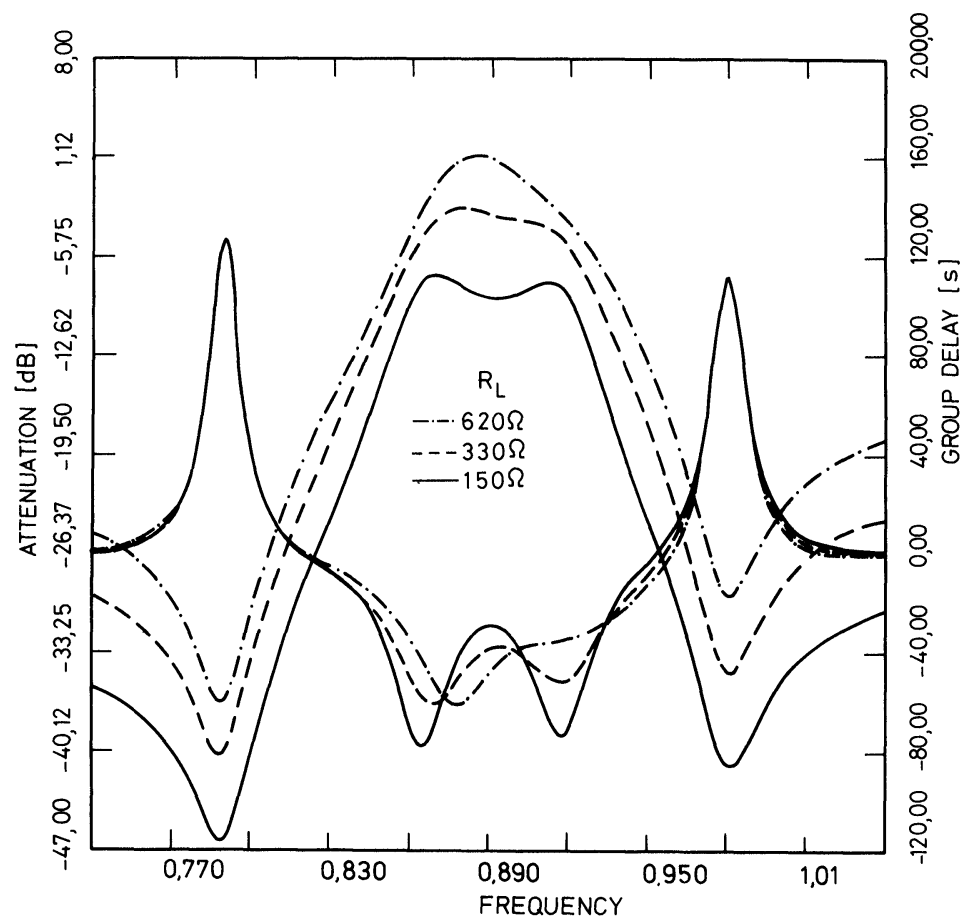

FIGURE 15 Calculated filter responses as the load impedance changes (a correcting factor of 0.25 had to be used for the load admittance value).

calculations and experimental results. It can be seen that as the normalized load conductance changes from 4 to 43 , the measured frequency change is only $0.8 \%$ less than the calculated change.

Figure 15 shows the calculated responses with a different load resistance (considerably larger than that of Table III) and in Figure 16, are the corres- ponding measured responses. When calculating these curves, a correcting factor of 0.25 has to be used for the value of load conductance. Since the curves correspond so well to each other it is possible to conclude that the equivalent circuits and the results that were achieved from them can be considered reliable.

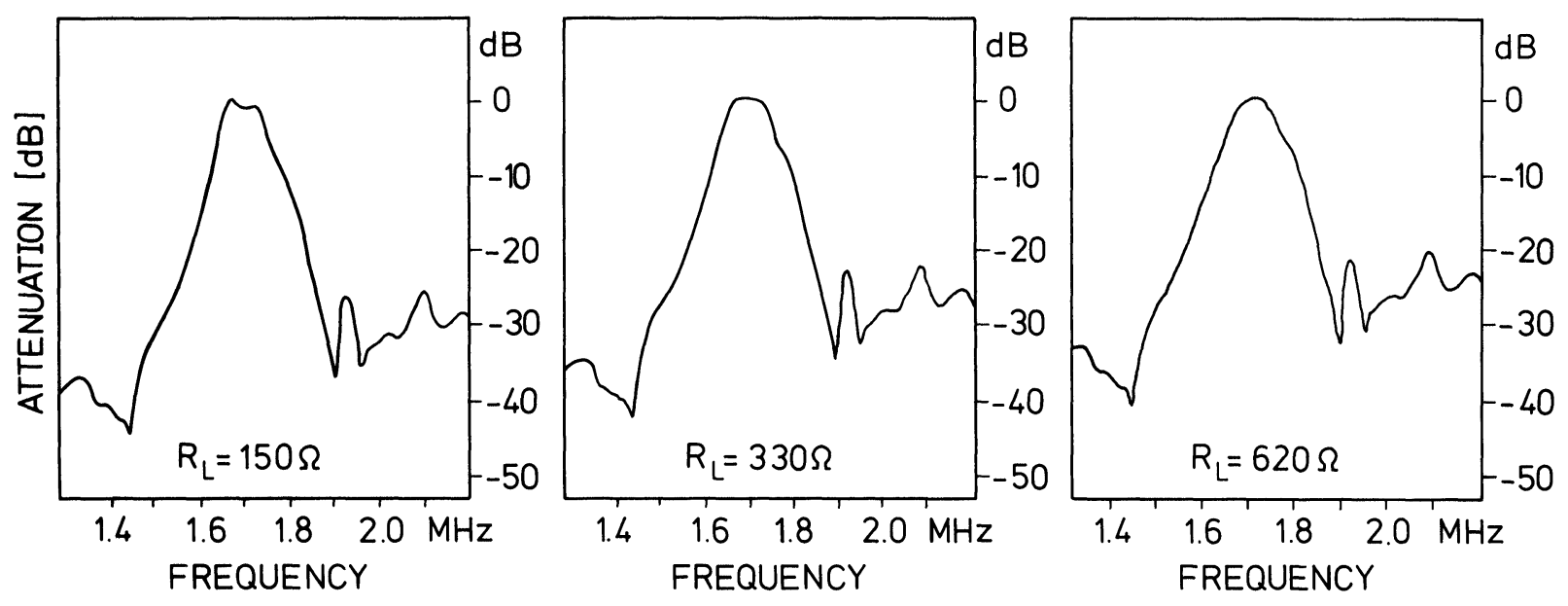

FIGURE 16 The measured filter responses of the filter whose calculated responses are shown in Figure 15. 
Although it is possible to reduce the number of resonances to the required number by applying the energy trapping phenomenon, it is almost inevitable that some other method will have to be used in order to keep the spurious resonances to a minimum. In commercial monolithic filters the ceramic plate is made thinner or even partly cut off between different resonators and the packaging material is used as an attenuation material at the sides of the ceramic plate. In the experiments epoxy cement was used as an energy absorbant material. The attenuation material also effects the $Q$-factor of the filter and its value can be adjusted by the amount of energy absorbant material applied.

Figure 17 shows a calculated electrode design for

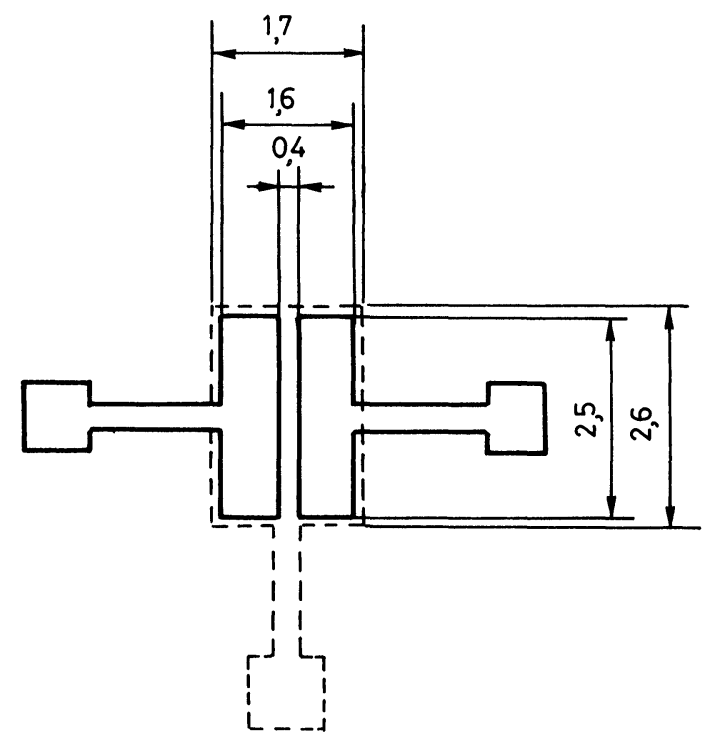

FIGURE 17 The dimensions of the electrodes of a 1.85 $\mathrm{MHz}$ filter.

a filter with a centre frequency of $1.85 \mathrm{MHz}$, a $-20 \mathrm{~dB}$ bandwidth of at least $200 \mathrm{kHz}$ and a phase response as linear as possible. The pattern has been evaporated onto a $0.52 \mathrm{~mm}$ thick polarized PZT-7A plate. By choosing a load impedance of $150 \Omega$ a filter with measured and calculated responses as shown in Figure 18 was constructed that confirms the reliability of this method.

\section{CONCLUSIONS}

In this paper the effects of different parameters on the response of a thickness-twist mode filter have been calculated. From measurements made from test filters it has been shown that the filter responses

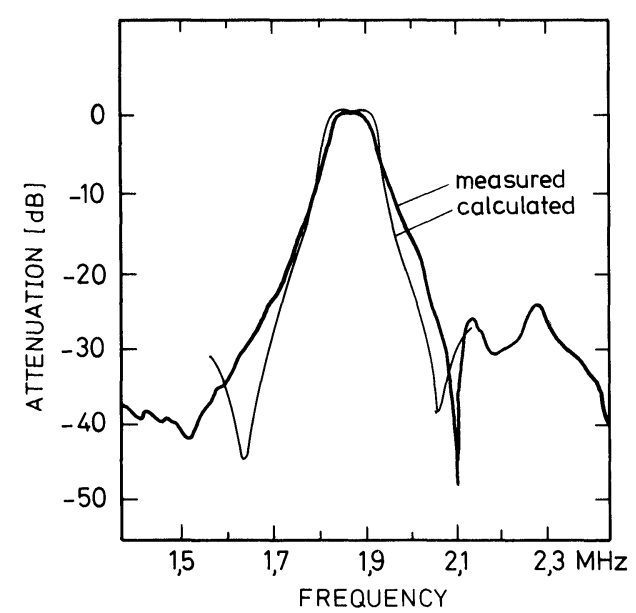

FIGURE 18 The calculated and measured filter responses of a $1.85 \mathrm{MHz}$ filter (Figure 16).

behave as predicted by the calculations. In order to design a filter the $Q$-factor must be measured from the test samples. From the calculations it is possible to design a filter with a centre frequency of 1 to $5 \mathrm{MHz}$, a bandwidth of 1 to $10 \%$, a load impedance of 100 to $600 \Omega$ and a characteristic phase response to meet the requirements. The 20 to $30 \mathrm{~dB}$. attenuation of the ceramic filter depends mainly upon the character of the ceramic material.

\section{REFERENCES}

1. R. Holland and E. P. EerNisse, Design of resonant piezoelectric devices. Research monograph No 56, The M.I.T. Press Cambridge, Massachusetts and London, England, pp. 179-210, 1967.

2. H. H. Schuessler, Ceramic filters and resonators, IEEE Transactions on Sonics and Ultrasonics, vol. 21, pp. 257-268, April 1974

3. M. Onoe and H. Jumonji, Analysis of piezoelectric resonators vibrating in trapped-energy modes. Electron. Commun Japan, vol. 48, pp. 84-93, Sept. 1965.

4. P. Wolfe, Capacitance calculations for several simple twodimensional geometries, Proceedings of the IEEE, No 50, pp. 2131-2132, October, 1962.

5. K. Nakamura and H. Shimizu, Equivalent circuits for thickness modes of elastic waves propagating in the plane of piezoelectric plate, Trans. IEEE Japan, vol. 55-A, pp. 95-102, Feb. 1972.

6. T. Uno, $200 \mathrm{MHz}$ thickness extensional mode $\mathrm{LiTaO}_{3}$ monolithic crystal filter, IEEE Trans. Sonics and Ultrasonics, vol. 22, pp. 168-174, May 1975.

7. Vernitron Ltd., Five modern piezoelectric ceramics, Bulletin 66011/F, Jan. 1976.

8. M. Onoe, H. Jumonji and N. Kobori, High Frequency crystal filters employing multiple mode resonators vibrating in trapped energy modes, Proc. 20th Feb. Control Symp., pp. 266-287, 1966. 

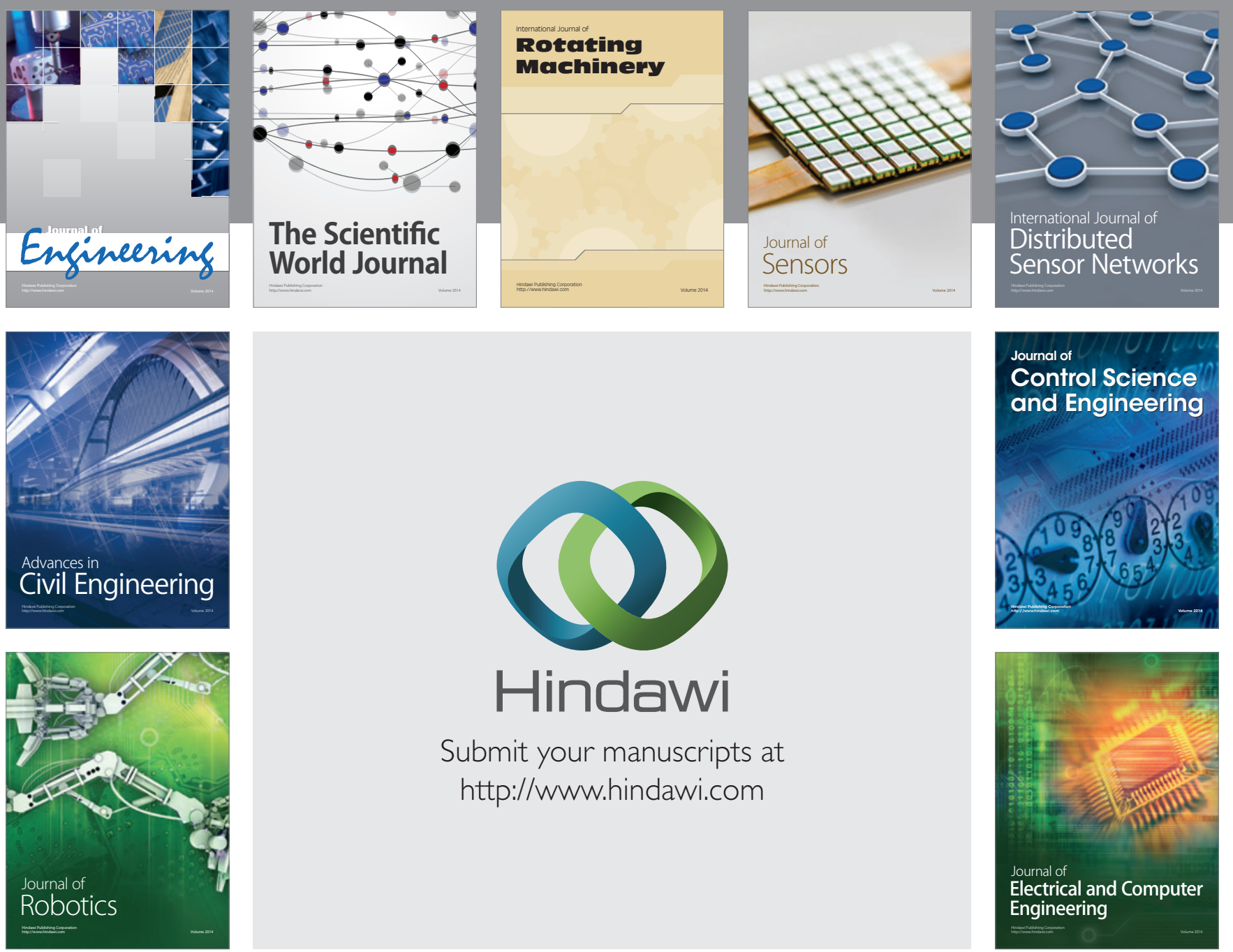

Submit your manuscripts at

http://www.hindawi.com
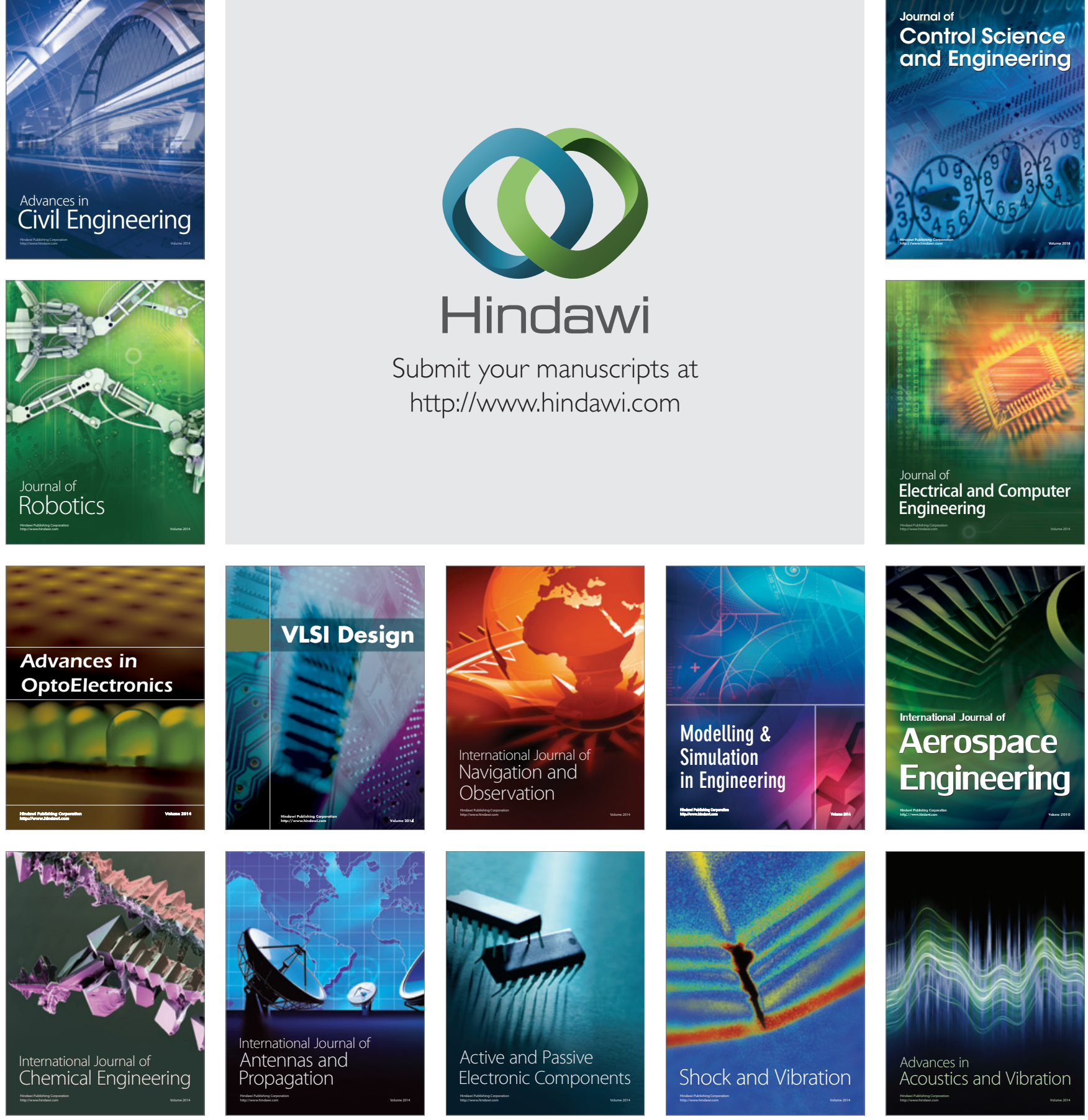